\title{
Lipoatrofia Facial Idiopática: A propósito de un caso
}

\author{
Idiopathic Facial Lipoatrophy: About a case
}

\author{
Yessenia Cruz Castillo. ${ }^{1}$, María José Pinos Cedeño. ${ }^{2}$, Alex Revelo. ${ }^{3}$ \& Johnny Steven \\ Valdez Benalcazar. ${ }^{4}$
}

Recibido: 04-08-2021 / Revisado: 16-08-2021 /Aceptado: 07-09-2021/ Publicado: 05-10-2021

DOI: https://doi.org/10.33262/anatomiadigital.v4i4.1887

\begin{abstract}
Resumen

Introduction: Facial lipoatrophy is a rare

Introducción: La lipoatrofia facial es una condition described by the anomalous condición rara descrita por la pérdida loss of subcutaneous fat that mainly affects the middle third of the face without compromising bone or muscle tissue. Lipoatrophy is not associated with exudative reactions or appreciable fibrosis. The etiology of lipoatrophy can be congenital or acquired, even traumatic, iatrogenic, or idiopathic. Generalized lipoatrophy is a rare disorder characterized by an almost complete absence of fat. Both congenital and anómala de la grasa subcutánea que afecta sobre todo al tercio medio facial sin compromiso de tejido óseo ni muscular. La lipoatrofia no se asocia con reacciones exudativas o fibrosis apreciable. La etiología de la lipoatrofia puede ser congénita o adquirida, incluso traumática, iatrogénica o idiopática. La lipoatrofia generalizada es un trastorno raro caracterizado por una casi completa ausencia de grasa. Tanto congénita como

\footnotetext{
${ }^{1}$ Médico Especialista en Medicina Interna y General del Ecuador, (Tungurahua/Ambato) Médico Internista del Hospital Regional Docente Ambato, Docente Universidad Técnica de Ambato. (D) ORCID ID: (http:// orcid.org/0000-0002-4642-885X), yessemallito@ hotmail.com

${ }^{2}$ Médico Especialista en Medicina Interna y General del Ecuador, (Tungurahua/Ambato) Médico Internista del Hospital Regional Docente Ambato, Docente Universidad Técnica de Ambato. (1D) ORCID ID: (http:// orcid.org/0000-0002-1146-6784)

${ }^{3}$ Médico Especialista en Imagenologia en la Universidad Estatal de Cuenca, (Tungurahua/Ambato), Hospital Regional Docente Ambato. (1) ORCID ID:(http:// orcid.org/0000-0003-0775-0501)

${ }^{4}$ Médico Cirujano en Universidad Regional Autónoma de los Andes "Uniandes", (Tungurahua/Ambato), (10) ORCID ID: (https://orcid.org/0000-0002-0766-4702)
} 
acquired. Objective: this report is to present a rare case of idiopathic facial lipoatrophy in a young patient without associated pathologies. Methodology: the research design was descriptive, it has only been previously identified in four case reports, among which the demographics of the patient vary considerably. Results: a case with the anatomical and paraclinical demographic characteristics of a rare case of Facial Lipodystrophy was described. Conclusion: it was concluded that idiopathic facial lipoatrophy that presents with a generalized absence of fat followed by a series of abnormalities such as insulin resistance and acanthosis nigricans before adolescence.

Keywords: facial lipoatrophy, lypodisthophy, idiopathic, facial adquirida. Objetivo: del presente informe es presentar un caso raro de lipoatrofia facial idiopática en paciente joven sin patologías asociadas. Metodología: el diseño de investigación fue de tipo descriptiva, solo se ha identificado previamente en cuatro informes de casos, entre los cuales, la demografía del paciente varía considerablemente. Resultados: se describió un caso con las características demográficas anatómicas y paraclínicas de un caso raro de Lipodistrofia Facial Conclusión: se concluyó que la Lipoatrofia facial idiopática que presenta con una ausencia generalizada de grasa seguido de una serie de anormalidades como resistencia a la insulina y acantosis nigricans antes de la adolescencia.

Palabras claves: lipoatrofia facial, lipodistròfia, idiopàtica, facial

\section{Introducción.}

La lipoatrofia es la desaparición de la grasa subcutánea, sin exudación o fibrosis apreciable. La lipoatrofia se divide en generalizada, parcial (extensa, pero no generalizada) y localizada (limitada a localizadas áreas). (Shalev, 2000).

No existe una razón universal o precisa comprobada para la lipoatrofia; sin embargo, la literatura sugiere en la patogénesis el deterioro de la diferenciación de adipocitos, apoptosis de adipocitos y disfunción mitocondrial. (Giralt, Domingo, \& Villarroya, 2011).

La lipoatrofia parcial fue reportada por primera vez en 1885 por Mitchell. Tiene un comienzo característico de pérdida progresiva de grasa subcutánea en la cara y el cuero cabelludo, y luego se extiende hasta las crestas ilíacas. El grupo objetivo son los niños de entre cinco y ocho años. (Anbarasi, Sathasivasubramanian, \& Krithika, 2012).

Las causas iatrogénicas de la lipoatrofia incluyen complicaciones de medicamentos inyectados como insulina, corticosteroides, antibióticos (penicilina G), hierro, heparina y vacunas. (Anbarasi, Sathasivasubramanian, \& Krithika, 2012)

Los pacientes VIH positivos en terapia antirretroviral, especialmente los inhibidores de la transcriptasa inversa nucleósidos (TRN) y los inhibidores de la proteasa (IP) 
manifiestan lipoatrofia como un efecto adverso del fármaco. (Giralt, Domingo, \& Villarroya, 2011)

El compromiso de la lipoatrofia facial incluye hundimiento de mejillas, pliegues profundos en la región nasolabial, depresión de la piel en las sienes, lados de la frente y alrededor de las cuencas de los ojos. La regresión espontánea en pocas semanas es habitual, sin embargo, en algunos casos la lesión puede persistir y causar problemas estéticos. (Anbarasi, Sathasivasubramanian, \& Krithika, 2012)

La lipoatrofia facial se puede clasificar de la siguiente manera. (Ascher, y otros, 2006).

Grado 0: sin lipoatrofia facial.

Grado 1: aplanamiento leve o sombreado en una o más regiones faciales; No hay marcas óseas prominentes ni visibilidad de la musculatura subyacente.

Grado 2: punto intermedio entre el grado 1 y el grado 3.

Grado 3: moderada concavidad de una o más regiones faciales, prominencia de marcas óseas y posible visibilidad de la musculatura subyacente.

Grado 4: punto intermedio entre el grado 3 y el grado 5.

Grado 5 - Depresión severa de una o más regiones faciales, prominencia severa de marcas óseas y visibilidad clara de la musculatura subyacente.

La tomografía computarizada (TC) desempeña un papel útil en el diagnóstico de la lipoatrofia focal ya que la grasa se puede apreciar fácilmente en la TC. El ultrasonido puede demostrar asimetría de la cara y pérdida de grasa, pero tienen menos sensibilidad que la TC. (Anbarasi, Sathasivasubramanian, \& Krithika, 2012)

\section{Descripción del caso}

Paciente femenina de 32 años de edad residente de Ambato-Ecuador, sin antecedentes clínicos de importancia y asintomática, que presenta hace 3 meses asimetría facial con disminución de densidad de hemicara izquierda por lo que acude. Niega uso de medicamentos, trauma facial, infecciones locales o alteración facial congénita. Además niega estas alteraciones en la familia.

Al examen físico hubo una pérdida casi completa de la almohadilla de grasa malar sobre la eminencia malar, así como una deformidad cóncava que se extendía temporalmente hacia el canto lateral. Fue evaluada clínicamente para tener concavidad moderada a severa, prominencia observable de hitos óseos y posible visibilidad de la musculatura subyacente. Estas características fueron consistentes con la lipoatrofia facial de grado 2 según la escala desarrollada por el Panel de Lipoatrofia Facial.

Se realizó estudios complementarios siendo los mismos normales incluyendo panel virológico e inmunológico. El estudio tomográfico se confirmó ausencia de compromiso 
óseo. La lipoatrofia facial fue considerablemente desfigurante y se evaluó como un Grado 2 en la escala de lipoatrofia facial.

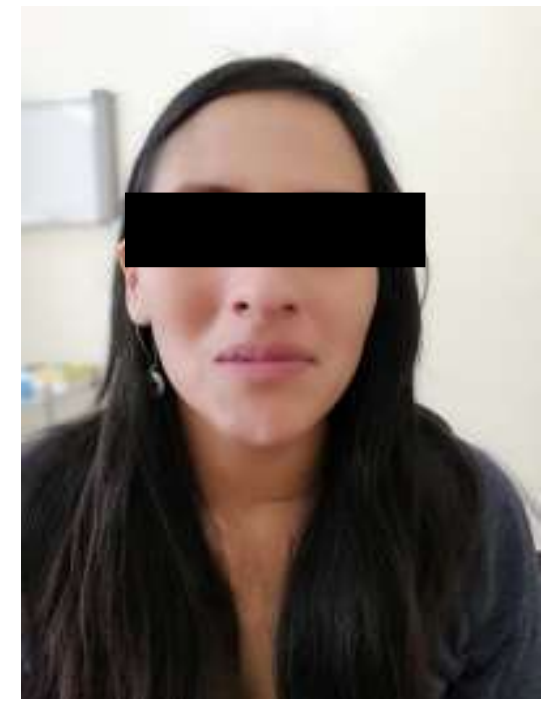

Figura 1. Lipoatrofia facial derecha, depresión de surco nasogeniano derecho

Fuente: Foto autorizada del paciente

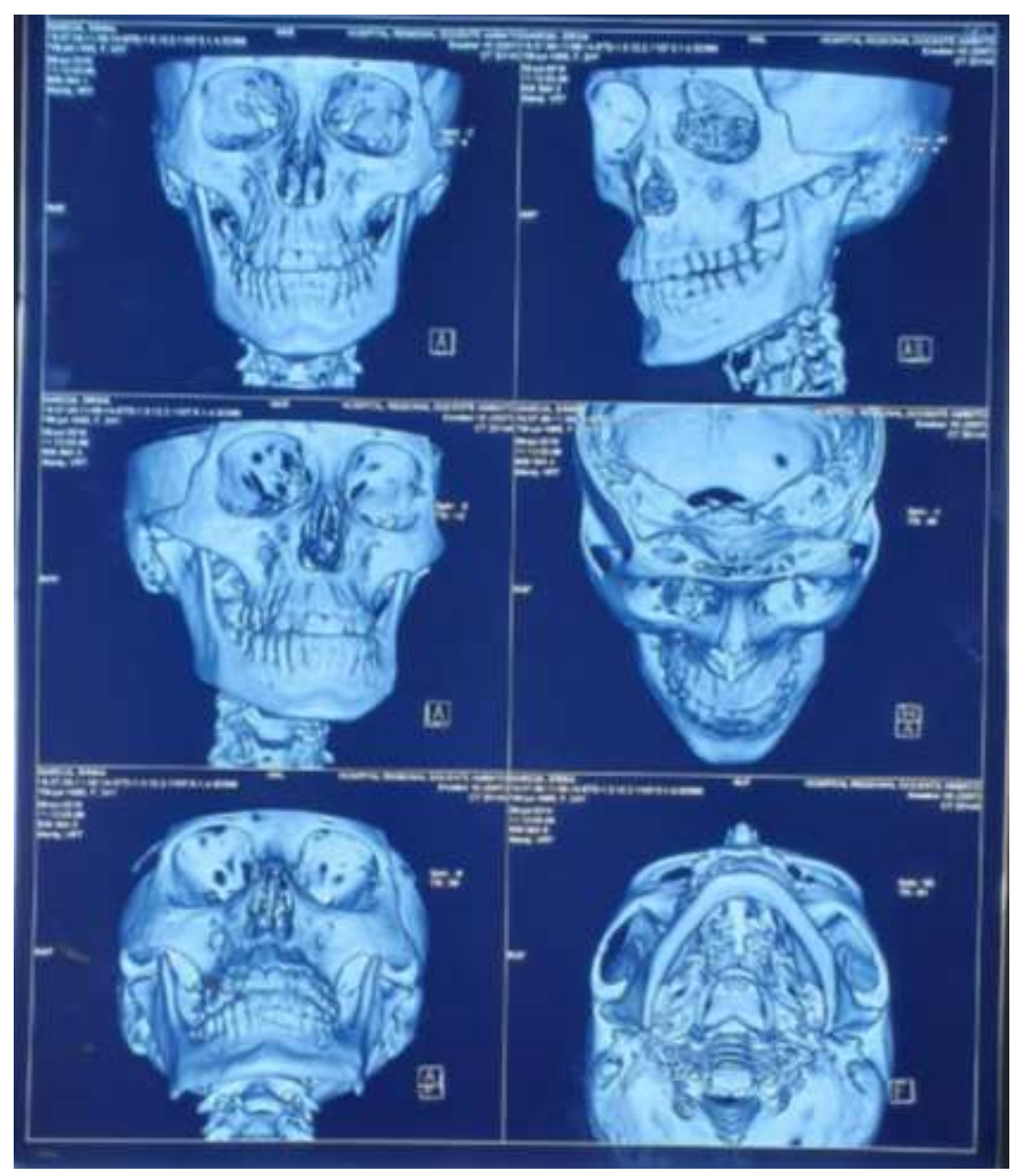

Figura 2. Macizo facial en la reconstrucción 3D no muestran lesiones intrínsecas o extrínsecas. Fuente: Historia clínica. 


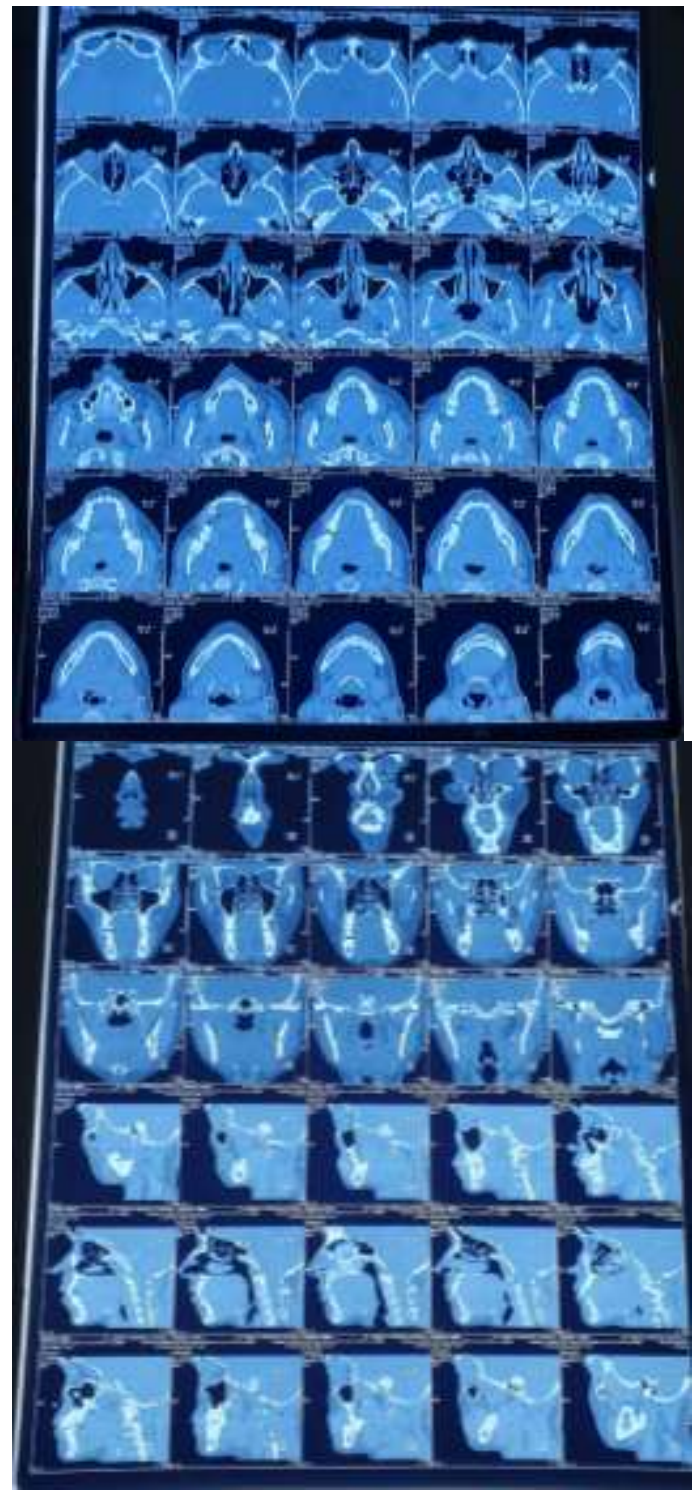

Figura 3. Tomografía simple cortes axiales y reconstrucciones coronales y sagitales. Asimetría de hemicara derecha dada por disminución severa del espesor del tejido celular subcutáneo, planos musculares presentan morfología y espesor normal. Glándulas Parótidas son simétricas, homogéneas. Ventana ósea no muestra lesiones blásticas, líticas o soluciones de continuidad, Adecuada neumatización de senos paranasales.

Fuente: historia clínica

\section{Discusión}

La lipodistrofia se refiere en general a los cambios en la producción, utilización y almacenamiento de grasa. La lipoatrofia se define por la desaparición de la grasa subcutánea (Anbarasi, Sathasivasubramanian, \& Krithika, 2012). La lipodistrofia se clasifica ampliamente en formas adquiridas y heredadas. Los tipos adquiridos se describen como lipodistrofia parcial generalizada o localizada asociada al VIH. Los tipos heredados incluyen lipodistrofia generalizada congénita tipos 1 y 2, lipodistrofia parcial familiar en la variedad Dunnigan o de mutaciones PPAR $\gamma$, o displasia mandibuloacral tipo A o B. (Abhimanyu Garg, 2006). 
La lipoatrofia facial describe el aplanamiento de los contornos normalmente convexos de la cara (Ascher, y otros, 2006) y las áreas más comúnmente afectadas son las mejillas, las sienes y la región preauricular, orbitaria o perioral (Aneta Szczerkowska-Dobosz, 2015). Se asocia con enfermedades hereditarias, enfermedades adquiridas y proceso de envejecimiento natural (Ascher, y otros, 2006) (Anbarasi, Sathasivasubramanian, \& Krithika, 2012). La etiología de la lipoatrofia facial es más comúnmente de naturaleza autoinmune, pero también se han identificado causas traumáticas (Groot, 1994). La lipoatrofia facial se ha descrito como una característica de otras afecciones. Las condiciones más comunes asociadas con la lipoatrofia facial son la terapia antirretroviral para la infección por VIH y los trastornos del tejido conectivo que están asociados con la paniculitis, como el lupus eritematoso y la esclerodermia localizada, también conocida como morfea (Anbarasi, Sathasivasubramanian, \& Krithika, 2012). La lipoatrofia iatrogénica generalmente es causada por inyecciones de medicamentos. La lipoatrofia facial idiopática es una condición rara que resulta en una desfiguración estética. Ha habido tres casos reportados previamente de lipoatrofia facial idiopática (Anbarasi, Sathasivasubramanian, \& Krithika, 2012) (Liu \& Cohen, 2010) (Tiago Pina Zanelato, 2013). El estigma y el efecto psicológico que esto puede tener en los pacientes tienen implicaciones potenciales significativas en la calidad de vida de los pacientes (Ascher, y otros, 2006).

En este caso, describimos a una mujer de 40 años sana que se presenta con lipoatrofia hemofacial idiopática grave de inicio agudo. La historia, el examen físico y los hallazgos en laboratorio se descartaron patologías asociadas.

Hasta la fecha, solo se han informado tres casos de lipoatrofia facial idiopática. Desafortunadamente, solo existe evidencia de baja calidad para la lipoatrofia facial idiopática porque la literatura está compuesta solo de informes de casos y revisiones. Todavía se desconoce mucho acerca de la lipoatrofia facial idiopática y los casos futuros deben documentarse en la literatura como un medio para caracterizar aún más este proceso de la enfermedad.

\section{Conclusión}

- La lipoatrofia facial idiopática es una rara afectación estética que provoca un estigma social y afecto directamente a la calidad de vida. Existen muy pocos casos reportados en la literatura, por lo que se desconoce varios aspectos epidemiológicos de esta enfermedad, por lo que la publicación de reporte de casos es de importancia para caracterizar este proceso idiopático.

\section{Referencias bibliográficas.}

Anbarasi K, Krithika C, Sathasivasubramanian S, VenkataSai P. Focal Lipoatrophy of face: a rare esthetic complaint. J Clin Imaging Sci. 2012;2:14. https://doi.org/10.4103/2156-7514.94229. 
Ascher B, Coleman S, Alster T, Bauer U, Burgess C, Butterwick K, et al. Full scope of effect of facial lipoatrophy: a framework of disease understanding. Dermatol Surg. 2006;32:1058-69.

Coleman SR, Katzel EB. Fat grafting for facial filling and regeneration. Clin Plast Surg. 2015;42:289-300.

Garg A. Acquired and inherited lipodystrophies. N Engl J Med. 2004;350:1220-35.

Groot ACDE. Is lipoatrophia semicircularis induced by pressure? $\mathrm{Br} \mathrm{J}$ Dermatol. 1994;131:887-90.

Liu SW, Cohen GF. Idiopathic hemi-facial lipoatrophy treated with autologous fat transfer. J Cosmet Dermatol. 2010;9:226-9.

Nagore E, Sánchez-motilla JM, Rodríguez-serna M, Vilata JJ, Aliaga A. Lipoatrophia semicircularis - a traumatic panniculitis: report of seven cases and review of the literature. J Am Acad Dermatol. 1998;39:879-81.

Rendon MI, Keeling J. Poly-L-lactic acid for the treatment of trauma-induced facial lipoatrophy and asymmetry. Cutis. 2008;81:218-22.

Szczerkowska-dobosz A, Olszewska B, Lemańska M, Purzycka-bohdan D, Nowicki R. Acquired facial lipoatrophy: pathogenesis and therapeutic options. Postepy Dermatol Alergol. 2015;32:127-33.

Zanelato TP, Marquesini G, Colpas PT, Magalhães RF, de Moraes AM. Implantation of autologous fat globules in localized scleroderma and idiopathic lipoatrophy report of five patients. An Bras Dermatol. 2013;88(6 suppl 1):1203. https://doi.org/10.1590/abd1806-4841.20132115

\section{LC Ciencia}




\section{PARA CITAR EL ARTÍCULO INDEXADO.}

Cruz Castillo, Y., Pinos Cedeño, M. J., Revelo, A., \& Valdez Benalcazar , J. S. (2021). Lipoatrofia Facial Idiopática: A propósito de un caso. Anatomía Digital, 4(4), 15-22. https://doi.org/10.33262/anatomiadigital.v4i4.1887

\section{LCiencia}

El artículo que se publica es de exclusiva responsabilidad de los autores y no necesariamente reflejan el pensamiento de la Revista Anatomía Digital.

El artículo queda en propiedad de la revista y, por tanto, su publicación parcial y/o total en otro medio tiene que ser autorizado por el director de la Revista Anatomía Digital.
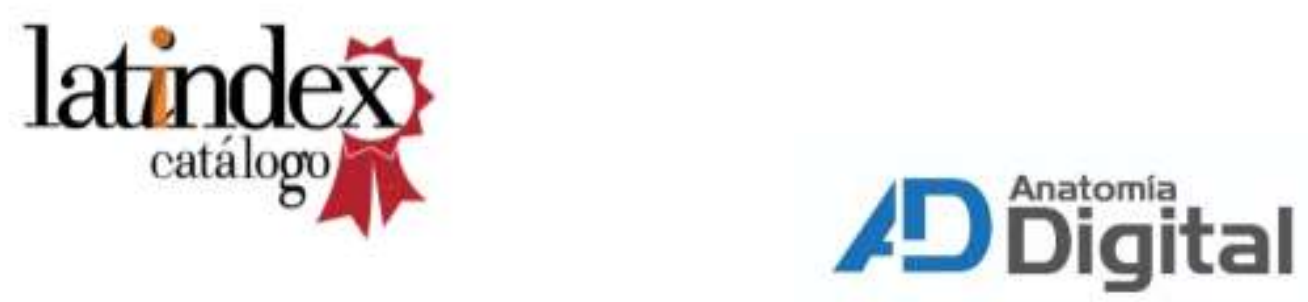